\title{
ПОЛИКРИТЕРИАЛЬНЫЙ ПОДХОД К АНАЛИЗУ МЕРОПРИЯТИЙ ПО ПОВЫШЕНИЮ УСТОЙЧИВОСТИ УЗЛОВ ЭЛЕКТРОДВИГАТЕЛЬНОЙ НАГРУЗКИ НЕФТЕДОБЫВАЮЩИХ ПРЕДПРИЯТИЙ
}

\author{
Лосев Федор Алексеевич', \\ fedor_los@mail.ru \\ Сушков Валерий Валентинович², \\ sushkovvv@gray-nv.ru \\ 1 Тюменский индустриальный университет,
Россия, 625000, г. Тюмень, ул. Володарского, 38.
${ }^{2}$ Нижневартовский государственный университет,
Россия, 628600, г. Нижневартовск, ул. Ленина, 56.
}

Актуальность. Работоспособность технологических систем нефтяного месторождения напрямую зависит от надежности электроснабжения. Наиболее частыми нарушениями электроснабжения являются провалы и прерывания напряжения, которые могут привести к потере устойчивости узлов электродвигательной нагрузки, что ведет к экономическим потерям нефтедобывающего предприятия. Для повышения устойчивости узлов нагрузки могут применяться различные устройства, например динамические компенсаторы искажений напряжения и источники бесперебойного питания, также устойчивость позволяет повысить правильная настройка устройств автоматического восстановления питания потребителей, однако при этом необходимо учитывать степень независимости источников питания.

Цель: разработать поликритериальный подход к анализу мероприятий по повышению устойчивости узлов электродвигательной нагрузки нефтедобывающих предприятий, учитывающий экономические и технические факторы.

объекты: узел электродвигательной нагрузки, устройства повышения устойчивости узлов нагрузки.

Методы: математическое моделирование работы узлов электродвигательной нагрузки при провалах и прерываниях напряжения; математическое моделирование устройств повышения устойчивости узлов нагрузки; оценка экономической эффективности мероприятий по повышению устойчивости узлов нагрузки.

Результаты. Для анализа мероприятий по повышению устойчивости узлов электродвигательной нагрузки предложен поликритериальный подход, позволяющий учитывать как экономические, так и технические факторы. Для оценки технических факторов предложено использовать коэффициент запаса устойчивости по напряжению и коэффициент зависимости источников питания, для оценки экономических факторов - коэффициент экономической эффективности капитальных вложений. Проведен анализ эффективности применения динамических компенсаторов искажений напряжения и быстродействующего автоматического ввода резерва для повышения устойчивости узлов нагрузки кустовых насосных станций и кустов скважин.

\section{Ключевые слова:}

Повышение устойчивости узлов нагрузки, граница динамической устойчивости, динамический компенсатор искажений напряжения, быстродействующий автоматический ввод резерва, кустовая насосная станция.

\section{Введение}

Нефтяное месторождение включает ряд взаимозависимых технологических систем, которые обеспечивают нефтедобычу, например, система добычи, сбора, подготовки и транспорта нефти, система поддержания пластового давления (ППД) [1]. Каждая технологическая система электрифицирована и зависит от работоспособности системы электроснабжения [2].

Одним из наиболее частых нарушений электроснабжения являются провалы и прерывания напряжения, которые могут привести к потери устойчивости узла электродвигательной нагрузки и к большому недоотпуску продукции и, соответственно, к экономическим потерям. Для предотвращения подобной ситуации применяют различные устройства для повышения устойчивости узла электродвигательной нагрузки, например, динамические компенсаторы искажений напряжения (ДКИН), источники бесперебойного питания
(ИБП) $[3,4]$ и т. д. ДКИН обладает высокой скоростью срабатывания, но время его работы ограничено. ИБП лишен технических ограничений ДКИН по времени работы, но имеет большую стоимость. В этом случае необходимо также учитывать степень независимости источников питания и надежность срабатывания устройств восстановления питания потребителей путем автоматического присоединения резервного источника питания при отключении рабочего источника питания, таких как автоматический ввод резерва $(\mathrm{ABP})$ и быстродействующий автоматический ввод резерва (БАВР). Таким образом, проблема повышения устойчивости узла электродвигательной нагрузки имеет два аспекта - технический и экономический, тогда разработка поликритериального подхода к анализу мероприятий по повышению устойчивости узлов электродвигательной нагрузки нефтедобывающих предприятий является актуальной. 


\section{Поликритериальный подход}

Экономический подход включает определение капитальных затрат на мероприятия по повышению устойчивости и экономического эффекта, вызванного этими мероприятиями.

Для оценки эффективности мероприятий по повышению устойчивости узлов нагрузки предлагается использовать коэффициент экономической эффективности капитальных вложений [5]:

$$
\mathrm{K}_{\ni}=\frac{\vartheta_{t}}{3},
$$

где 3 - капитальные вложения в мероприятия по повышению устойчивости; $Э_{t}-$ экономический эффект от повышения устойчивости, достигаемый в течение 1 года.

Капитальные вложения в мероприятия по повышению устойчивости:

$$
3=3_{i}-3_{\sigma},
$$

где $3_{6}$ - капитальные вложения при базовом варианте электроснабжения; $3_{i}$ - капитальные вложения при предлагаемом варианте электроснабжения с повышением устойчивости узлов электродвигательной нагрузки.

Потеря устойчивости узла электродвигательной нагрузки приводит к полному или частичному простою потребителей, что ведет к недоотпуску продукции (нефти), простою основных фондов. Для устранения негативных последствий простоя применяют форсированные режимы работы технологического оборудования (включение резервных насосных агрегатов для выполнения плана по закачке воды в нефтяной пласт, например, на кустовых насосных станциях (КНС)). Сокращение времени форсировки ведет к снижению повышенного потребления электроэнергии [2] за время форсирования режима работы насосных агрегатов. Таким образом, можно принять, что экономический эффект в данном случае будет равен величине снижения экономического ущерба при проведении мероприятий по повышению устойчивости узла электродвигательной нагрузки. В общем виде примем, что экономический эффект имеет следующие составляющие: снижение упущенной прибыли из-за недоотпуска продукции $Э_{t 1}$, уменьшение потребления электроэнергии за счет работы оборудования в форсированных режимах $Э_{t 2}$ и снижение условнопостоянных расходов в себестоимости нефти $\vartheta_{t 3}$ :

$$
\vartheta_{t}=\vartheta_{t 1}+\vartheta_{t 2}+\vartheta_{t 3}
$$

Снижение упущенной прибыли из-за недоотпуска продукции определяется разностью ущербов от остановки технологии при базовом и предлагаемом вариантах электроснабжения (с устройствами по повышению устойчивости). При строительстве новых объектов за базовый вариант принимаются типовые схемы подстанций, типовое оборудование. При модернизации объектов базовым вариантом выступает существующая система электроснабжения. Тогда первая составляющая экономического эффекта равна

$$
\ni_{t 1}=\mathrm{y}_{1 \sigma}-\mathrm{y}_{1 i},
$$

где $\mathrm{y}_{16}$ - ущерб от остановки производства при базовом варианте электроснабжения без устройств по повышению устойчивости; $\mathrm{V}_{1 i}$ - ущерб от остановки производства при предлагаемом варианте электроснабжения с устройствами по повышению устойчивости узлов электродвигательной нагрузки.

Тогда ущерб, обусловленный потерей прибыли нефтедобывающего предприятия за год, равен

$$
\mathrm{y}_{1}=Q\left(Ц-\mathrm{C}_{\mathrm{H}}\right),
$$

где Ц - оптовая цена нефти, $\mathrm{p} / \mathrm{m}^{3} ; \mathrm{C}_{\text {н }}$ - себестоимость нефти, $\mathrm{p} / \mathrm{m}^{3}$

Годовые потери продукции, вызванные потерей устойчивости узлов нагрузки, определяются [6]:

- для насосных скважин

$$
Q=t_{\text {п }} \sum_{i=1}^{n} q_{i},
$$

где $t_{\text {п }}$ время простоя за год по причине потери устойчивости, ч; $q_{i}$ - дебит отдельных скважин по нефти, $\mathrm{M}^{3} /$ ч; $n$ - количество эксплуатационных скважин.

- для КНС, водозабора

$$
Q=\frac{Q_{\Sigma} t_{\text {пा }}\left(1-b_{\mathrm{cp}}\right)}{K_{\text {сс }} K},
$$

где $b_{\text {ср }}$ - средняя обводненность продукции по способам эксплуатации; $K_{\text {зс }}$ коэффициент учета замороженных скважин; $K$ - отношение объема закачиваемой воды к объему добываемой жидкости.

Экономический эффект, обусловленный снижением потребления электроэнергии за счет уменьшения времени работы оборудования в форсированных режимах, определяется разницей затрат на электрическую энергию при базовом и предлагаемом вариантах электроснабжения:

$$
\vartheta_{t 2}=3_{1 \sigma}-3_{1 i}
$$

где $3_{10}$ - затраты на электроэнергию в форсированных режимах работы оборудования при базовом варианте электроснабжения; $3_{1 i}$ - затраты на электроэнергию в форсированных режимах работы оборудования при предлагаемом варианте электроснабжения с повышением устойчивости узлов электродвигательной нагрузки.

Расчет потребления электроэнергии, например для КНС, основан на определении продолжительности работы насосного блока в форсированном режиме по следующей методике [6]:

1. Время простоя за год

$$
t_{\text {пा }}=T_{\text {пр }} N \text {, }
$$

где $N$ - ожидаемое количество отключений КНС; $T_{\text {пр }}$ - время простоя при отключении КНС.

2. Потери продукции при закачке, перекачке

$$
Q_{\text {прод }}=Q_{\Sigma} t_{\text {п }} \text {, }
$$

где $Q$ - суммарная производительность насосного блока в нормальном режиме.

3. Производительность насосного блока при включении резервных насосов в послеаварийном режиме 


$$
Q_{\text {п.а }}=Q_{\text {н }}\left(n_{\text {осн }}+K_{\text {и }} n_{\text {рез }}\right) K_{\text {пр }},
$$

где $Q_{\text {н }}$ - производительность насоса в нормальном режиме; $n_{\text {осн }}-$ число рабочих насосов; $n_{\text {рез }}-$ число резервных насосов; $K_{\text {и }}$ коэффициент использования резервных насосов; $K_{\text {пр }}$ - коэффициент, учитывающий снижение производительности насоса при включении дополнительных агрегатов.

4. Продолжительность работы насосного блока для ликвидации последствий отключения КНС

$$
t_{\text {п.а }}=\frac{Q_{\text {прод }}}{Q_{\text {п.а }}-Q_{\Sigma}} .
$$

5. Дополнительные энергозатраты

$$
W=K_{\text {и }} n_{\text {рез }} P_{\text {н }} K_{3} t_{\text {п.а }},
$$

где $P_{\text {н }}$ - номинальная мощность электродвигателей насосов; $K_{3}$ - коэффициент загрузки электродвигателей насосов.

Далее определяются экономические затраты на электроэнергию, например, для двухставочного тарифа

$$
3_{1}=W\left(\frac{\alpha}{T_{\mathrm{M}}}+\beta\right),
$$

где $\alpha$ - тариф за максимум заявленной мощности, $\mathrm{p} /$ кВт; $\beta$ - тариф за потребленную электроэнергию, $\mathrm{p} /$ кВт.ч; $T_{\text {м }}$ число часов использования максимума нагрузки, ч/год.

Экономический эффект от снижения условнопостоянных расходов в себестоимости нефти будет намного меньше, чем эффект от снижения упущенной прибыли и эффект от снижения потребления электроэнергии в форсированных режимах работы. Уменьшение времени простоя оборудования из-за потери устойчивости узлов нагрузки позволит уменьшить условно-постоянные расходы, но они в большей степени обусловлены следующими факторами: амортизация скважин и прочих основных средств, цеховые и общепромысловые расходы, заработная плата, расходы на освоение и подготовку новых скважин [7]. Таким образом, при оценке экономического эффекта от повышения устойчивости узлов нагрузки нефтедобывающих предприятий пренебрегаем снижением условно-постоянных расходов в себестоимости нефти из-за его малого влияния на общий экономический эффект.

Мероприятия по повышению устойчивости представляют собой инвестиционной проект, одним из основных факторов которого является дисконтированный срок окупаемости. Это период возврата денежных средств с учетом временной стоимости денег (ставки дисконта) [7]. В электроэнергетике дисконтированный срок окупаемости для эффективных проектов составляет 5-8 лет [8]. При повышении устойчивости узлов электродвигательной нагрузки экономический эффект достигается за счет сокращения времени простоя оборудования из-за аварийных отключений, возникающих при потере устойчивости $[9,10]$. Таким образом, выражение для определения экономическо- го эффекта повышения устойчивости узлов нагрузки с учетом дисконтирования имеет вид [7]:

$$
\ni=\sum_{t=1}^{T}\left(\vartheta_{t}-3_{t}\right) \frac{1}{(1+E)^{t}},
$$

где $\vartheta_{t}-$ экономический эффект в $t$-м году; $3_{t}-$ затраты, осуществляемые в $t$-м году; $T$ - количество лет; $E$ - ставка дисконта.

Для объектов электроэнергетики затраты в первом расчетному году состоят из капитальных и эксплуатационных затрат, в последующие годы остаются только эксплуатационные затраты, которые включают расходы на ремонт и обслуживание оборудования, а также амортизационные отчисления. Согласно [8], ежегодные издержки на ремонты и обслуживание элементов электрической сети напряжением 6-35 кВ составляют 4 \% капитальных затрат, амортизационные отчисления для электрических сетей напряжением 6-35 кВ в среднем составляют $6 \%$. С учетом вышесказанного упростим выражение (2):

$$
\vartheta=-3+\sum_{t=1}^{T}\left(\vartheta_{t}-0,13\right) \frac{1}{(1+E)^{t}} .
$$

Подставив (1) в (3) и упростив, получаем выражение для экономического эффекта повышения устойчивости, включающее только капитальные вложения и коэффициент их экономической эффективности:

$$
\ni=-3+3 \sum_{t=1}^{T}\left(K_{\ni}-0,1\right) \frac{1}{(1+E)^{t}} .
$$

Для определения граничных значений коэффициента экономической эффективности капитальных вложений в мероприятия по повышению устойчивости узлов нагрузки ставка дисконта принималась равной 0,1 , дисконтированный срок окупаемости 5 лет:

$$
\left\{\begin{array}{l}
\vartheta=-3+3 \sum_{t=1}^{5} K\left(K_{\ni}-0,1\right) \frac{1}{1,1^{t}} ; \\
\vartheta>0 .
\end{array}\right.
$$

Далее раскрыли знак суммы и получили выражение:

$$
-3+3\left(K_{э}-0,1\right) \cdot 3,79>0 .
$$

После математических преобразований получено следующее неравенство

$$
K_{\ni}>0,364 .
$$

Таким образом, при значении коэффициента экономической эффективности капитальных вложений больше 0,364 мероприятия по повышению устойчивости узлов нагрузки окупятся за срок не более 5 лет при ставке дисконта 0,1 , следовательно, их можно рекомендовать к внедрению.

Технический подход включает в себя определение ожидаемого годового числа отключений потребителей, вызванных провалами и прерываниями напряжения, с учетом коэффициента зависимости источников питания. Ожидаемое количество про- 
валов и прерываний напряжения, приводящих к потере устойчивости, определяется следующим образом:

1. Определяется ГДУ выбранного узла электродвигательной нагрузки с учетом несимметричных провалов напряжения [11]. Для этого составляется схема замещения в неподвижной относительно ротора электродвигателя системе координат $d-q$, состоящая из: схем замещения электродвигателей, схем замещения линий электропередачи, схем замещения трансформаторов и схемы замещения внешней системы электроснабжения [12-14]. Для оценки влияния несимметричных провалов напряжения производится их разложение на симметричные составляющие, затем моделируется выбег электродвигателей под воздействием напряжения прямой и обратной последовательностей в течение длительности провала напряжения. Далее моделируется самозапуск электродвигателей при восстановлении напряжения системы до номинального значения. Если самозапуск успешный, то увеличивается длительность провала напряжения, если неуспешный, то значение длительности и глубины определяют точку на ГДУ и цикл повторяется.

2. Определяется ожидаемое годовое количество отключений потребителей, вызванных провалами и прерываниями напряжения с заданным распределением их количества в координатах глубины и длительности на основе метода [15]. Для оценки используется статистическое распределение провалов и прерываний напряжения для смешанных (кабельных и воздушных) сетей, приведенное в ГОСТ 32144 [16], также может использоваться распределение провалов и прерываний напряжения, полученное на основе измерений на рассматриваемом энергетическом объекте. Полученное распределение строится в виде поверхности нормированного среднегодового количества возмущений, затем рассчитывается объем, ограниченный поверхностью нормированного количества возмущений и плоскостями времени ввода резерва и кривой динамической устойчивости.

3. Коэффициент зависимости источников определяется по методике, описанной в [17]. На первом этапе создается математическая модель сети внешнего электроснабжения, затем во всех узлах схемы моделируются трехфазные короткие замыкания и фиксируются значения остаточных напряжений на шинах низшего напряжения рассматриваемого узла электродвигательной нагрузки. Далее определяются узлы, короткие замыкания в которых привели к глубоким провалам напряжения (остаточное напряжение меньше напряжения статической устойчивости узла нагрузки) на первой секции шин, на второй секции шин и на двух секциях шин одновременно. На следующем этапе по параметрам потока отказа линий электропередачи определяется количество отказов для каждого узла электрической сети внешнего электроснабжения и рассчитывается коэффициент зависимости источников питания по выражению:

$$
K_{3}=\frac{N_{1,2}}{N_{1}+N_{2}-N_{1,2}},
$$

где $N_{1}$ - число критических провалов напряжения на первом ИП за время наблюдения $T ; N_{2}$ - число критических провалов напряжения на втором ИП за время наблюдения $T$; $N_{1,2}$ - число одновременных критических провалов напряжения на обоих ИП за время наблюдения $T$.

4. Учитывается влияние степени независимости источников питания на ожидаемое годовое количество отключений потребителей из-за потери устойчивости узла электродвигательной нагрузки. Коэффициент зависимости $K_{3}$ представляет собой вероятность провала напряжения на двух источниках питания одновременно [17], следовательно, чем меньше коэффициент зависимости источников, тем больше вероятность срабатывания АВР и БАВР, и наоборот. Также для непрерывных производств коэффициент зависимости источников питания относительно трехфазных коротких замыканий не должен превышать 0,5 [17].

При применении на подстанции БАВР возможны ситуации, при которых все виды провалов напряжения устраняются за счет переключения на резервный источник за доли секунды, тогда ожидаемое количество отключений потребителя из-за потери устойчивости, определенное по многомерному показателю устойчивости, будет равно нулю. Это условие выполнится только в случае стопроцентного срабатывания БАВР и при полной независимости источников питания, что на практике не встречается.

Таким образом, с учетом независимости источников питания ожидаемое годовое количество отключений потребителей, вызванных провалами напряжения с заданным распределением их количества в координатах глубины и длительности, предлагается использовать следующее выражение:

$$
N=\left\{\begin{array}{l}
N_{0} K_{3}, \text { если } N_{\text {п }}=0 \text { или } K_{3}>0,5 ; \\
\frac{N_{\text {п }}}{1-K_{3}}, \text { если } K_{3} \leq 0,5,
\end{array}\right.
$$

где $N_{0}$ - ожидаемое количество провалов напряжения, приводящих к нарушению устойчивости узлов нагрузки, в системе электроснабжения потребителя согласно статистическим данным; $N_{\text {п }}$ ожидаемое количество отключений потребителей, рассчитанное по многомерному показателю устойчивости.

\section{Результаты экспериментов}

Для апробации предложенного поликритериального подхода были выбраны два устройства для повышения устойчивости узлов электродвигательной нагрузки: БАВР и ДКИН, которые являются наиболее эффективными техническим решениями для объектов нефтедобычи [18].

Для оценки влияния данных устройств на узел электродвигательной нагрузки использовался ко- 


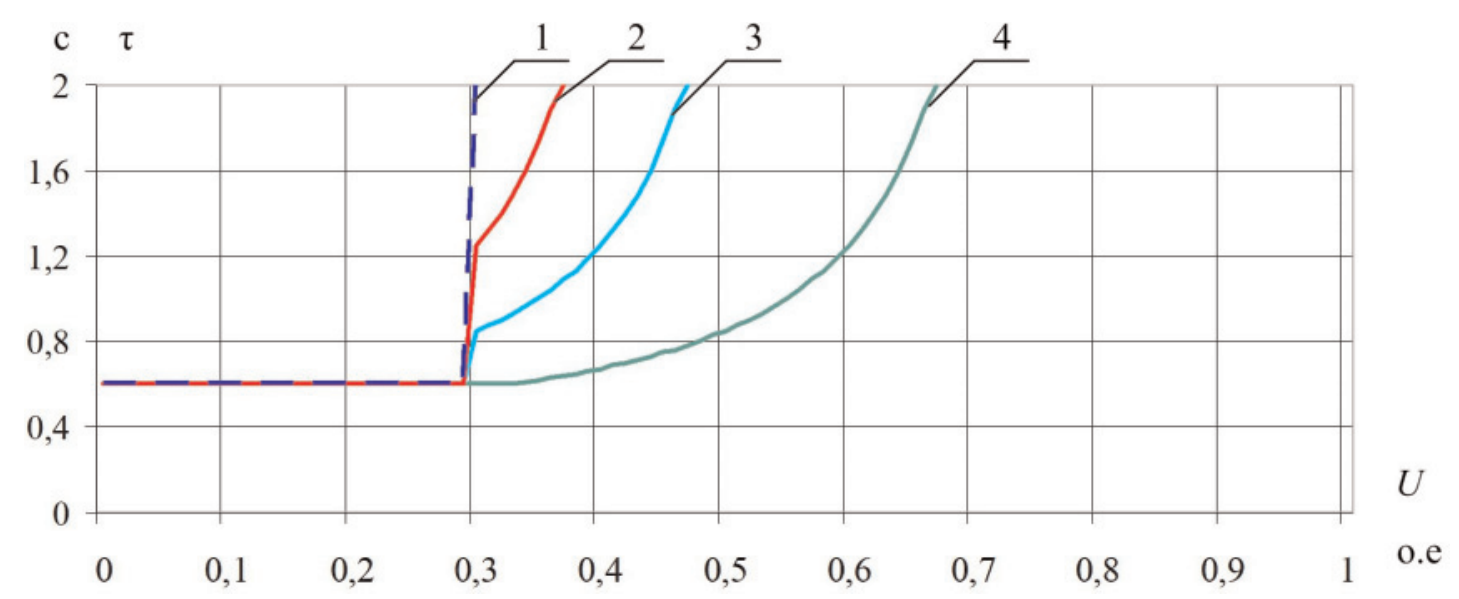

Pис. 1. Границы динамической устойчивости узла нагрузки КНС с электродвигателями серии АРМ мощностью 1250 кBт, напряжением 6 кВ при использовании: 1) ДКИН 40\%, 2) ДКИН $30 \%$, 3) ДКИН 20\%, 4) без ДКИН

Fig. 1. Dynamic stability curves of the sectional pump station with electric motors ARM series with power of $1250 \mathrm{~kW}$, voltage of $6 \mathrm{kV}$ when using: 1) DVR $40 \%$,2) DVR $30 \%$, 3) DVR 20\%,4) without DVR

эффициент запаса устойчивости по напряжению, который определяется по выражению [19]:

$$
K_{\text {зу }}=\frac{S_{\text {уст }}}{S_{\text {общ }}}
$$

где $S_{\text {усг }}$ - площадь области устойчивой работы, ограниченная границей динамической устойчивости и единицей по оси напряжения; $S_{\text {обш }}$ - площадь области, ограниченная единицей по оси напряжения и временем ввода резерва $t_{\text {пр }}$ по оси длительности провала напряжения.

ДКИН обеспечивает компенсацию провалов напряжения глубиной до $70 \%$ длительностью до 30 секунд, при этом данные устройства отличаются по степени компенсации провалов напряжения от 20 до $60 \%$ от номинального значения [20-25]. Для определения наиболее эффективного ДКИН по степени компенсации было проведено моделирование для узла нагрузки КНС с электродвигателями серии АРМ мощностью 1250 кВт напряжением 6 кВ и получены ГДУ (рис. 1).

В качестве электрической сети внешнего электроснабжения использовалась схема питания потребителей первой категории по надежности электроснабжения, включающая подстанции напряжением $110 / 35 / 6$ кB и $35 / 6$ кB, коэффициент зависимости источников для которой равен 0,29. Ожидаемое количество провалов и прерываний напряжения на шинах узла нагрузки было принято равным 4, распределение провалов было принято согласно ГОСТ 32144 [16]. На основании принятых данных был проведен анализ применения ДКИН для повышения устойчивости узла нагрузки КНС (табл. 1).

Для типовой схемы электроснабжения КНС коэффициент запаса устойчивости по напряжению равен 0,6 , при применении ДКИН $20 \%$ коэффициент запаса повысился до 0,73, при ДКИН 30 \% до 0,78 и при ДКИН $40 \%$ - до 0,8 (табл. 1). Дальнейшее увеличение глубины компенсации не дает роста запаса устойчивости по напряжению, так как рассматриваемые ДКИН не работают при остаточных напряжениях менее $30 \%$ [20-25]. Для каждого типа ДКИН был рассчитан коэффициент экономической эффективности капитальных вложений, а также были построены графики ущерба, обусловленного упущенной прибылью предприятия, и капитальных затрат (рис. 2) относительно базового значения, принятого равным 70 млн р.

Таблииа 1. Анализ приленения ДКИН для повышения устойчивости узла нагрузки КНС с электродвигателяли серии АРМ мощностью 1250 кBm, напряжением $6 \kappa B$

Table 1. Analysis of DVR using for increasing electromotive loading stability of the sectional pump station with electric motors ARM series with power of $1250 \mathrm{~kW}$ voltage of $6 \mathrm{kV}$

\begin{tabular}{|c|c|c|c|}
\hline $\begin{array}{c}\text { Тип устройства } \\
\text { Device type }\end{array}$ & $\begin{array}{c}\text { Коэффициент } \\
\text { запаса устойчи- } \\
\text { вости по напря- } \\
\text { жению } \\
\text { Voltage stability } \\
\text { reserve } \\
\text { coefficient }\end{array}$ & $\begin{array}{c}\text { Коэффициент } \\
\text { зависимости } \\
\text { источников } \\
\text { питания } \\
\text { Power supplies } \\
\text { dependence co- } \\
\text { efficient }\end{array}$ & $\begin{array}{c}\text { Коэффициент } \\
\text { экономической } \\
\text { эффективности } \\
\text { капитальных } \\
\text { вложений } \\
\text { Investment effici- } \\
\text { ency coefficient }\end{array}$ \\
\hline $\begin{array}{c}\text { Без ДКИН } \\
\text { Without DVR }\end{array}$ & 0,6 & 0,29 & - \\
\hline $\begin{array}{c}\text { ДКИН/DVR } \\
20 \%\end{array}$ & 0,73 & 0,29 & 0,91 \\
\hline $\begin{array}{c}\text { ДКИН/DVR } \\
30 \%\end{array}$ & 0,78 & 0,29 & 0,64 \\
\hline $\begin{array}{c}\text { ДКИН/DVR } \\
40 \%\end{array}$ & 0,8 & 0,29 & 0,49 \\
\hline
\end{tabular}

Согласно результатам расчета (табл. 1), для ДКИН 20 \% коэффициент экономической эффективности капитальных вложений равен 0,91 , для ДКИН $30 \%$ - 0,64, для ДКИН $40 \%$ - 0,49. Данная зависимость связана со значительным ростом капитальных вложений при увеличении глубины компенсации ДКИН, вызывающей незначительное снижение ущерба. Таким образом, наиболее эффективной степенью компенсации ДКИН явля- 


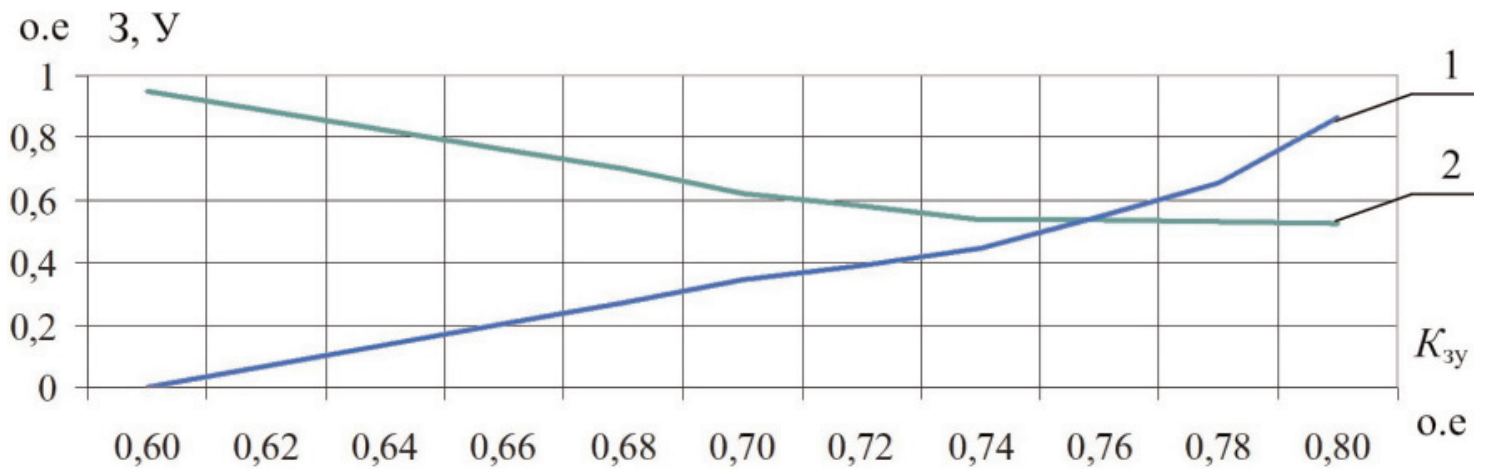

Pис. 2. 1) Зависилость капитальных затрат от коэффициента запаса устойчивости по напряжению при использовании ДКИН; 2 ) зависилость ущерба при потере устойчивости узла нагрузки от коэффициента запаса устойчивости по напряжению при использовании ДКИН

Fig. 2. 1) Capital investments dependences on voltage stability reserve coefficient when using DVR; 2) loss loading stability economic damage dependences on voltage stability reserve coefficient when using DVR

ется 20 \% для КНС с электродвигателями серии AРМ мощностью 1250 кВт напряжением 6 кВ.

Далее рассматривались варианты установки БАВР и ДКИН 20 \% на узел нагрузки КНС различной производительности и на узел нагрузки добывающих скважин с установками электрических центробежных насосов (УЭЦН) различной производительности. Для повышения точности моделирования погружного асинхронного электродвигателя можно использовать программно-аппаратные комплексы мониторинга параметров УЭЦН $[26,27]$. Установка БАВР на узел нагрузки позволяет устранять все виды провалов и прерываний напряжения за счет быстрого переключения на резервный источник питания, при этом коэффициент запаса устойчивости по напряжению равен 1. Для оценки эффективности ДКИН использовались ГДУ КНС (рис. 1) и кустов скважин (рис. 3). Схема внешнего электроснабжения и распределения провалов напряжения по длительности и глубине была принята неизменной для всех расчетов. Для узлов нагрузки КНС и кустов скважин были рассчитаны технические и экономический показатели мероприятий по повышению устойчивости (табл. 2).
Таблица 2. Анализ мероприятий по повышению устойчивости узлов электродвигательной нагрузки объектов нефтедобывающего предприятия

Table 2. Analysis of actions for increasing electromotive loading stability of the oil-extracting enterprises facilities

\begin{tabular}{|c|c|c|c|c|c|}
\hline 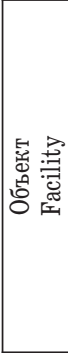 & $\begin{array}{c}\text { Производи- } \\
\text { тельность } \\
\text { Productivity }\end{array}$ & 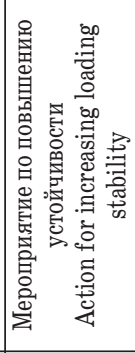 & 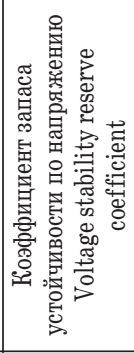 & 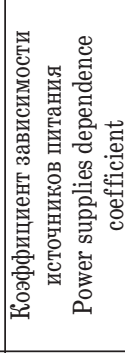 & 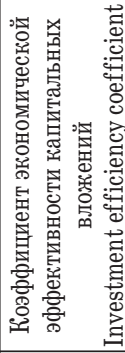 \\
\hline \multirow{3}{*}{ 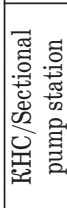 } & $Q=200 \mathrm{M}^{3} / \mathrm{\Psi}$ & \multirow{6}{*}{ 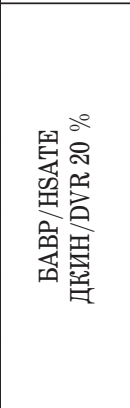 } & $\begin{array}{c}1 \\
0,73\end{array}$ & \multirow{6}{*}{0,29} & $\begin{array}{l}0,58 \\
0,59\end{array}$ \\
\hline & $Q=720 \mathrm{M}^{3} / \mathrm{\Psi}$ & & $\begin{array}{c}1 \\
0,73\end{array}$ & & $\begin{array}{l}1,74 \\
0,91 \\
\end{array}$ \\
\hline & $Q=1890 \mathrm{M}^{3} / \mathrm{\varphi}$ & & $\begin{array}{c}1 \\
0,73\end{array}$ & & $\begin{array}{l}2,23 \\
0,95\end{array}$ \\
\hline \multirow{3}{*}{ 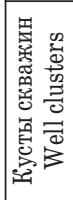 } & $Q=0,5 \mathrm{~T} / \mathrm{ч}$ & & $\begin{array}{c}1 \\
0,54\end{array}$ & & $\begin{array}{l}0,01 \\
0,03\end{array}$ \\
\hline & $Q=2,5 \mathrm{~T} / \mathrm{ч}$ & & $\begin{array}{c}1 \\
0,54\end{array}$ & & $\begin{array}{c}0,07 \\
0,1\end{array}$ \\
\hline & $Q=5 \mathrm{~T} / \mathrm{ч}$ & & $\begin{array}{c}1 \\
0,54\end{array}$ & & $\begin{array}{l}0,15 \\
0,19\end{array}$ \\
\hline
\end{tabular}

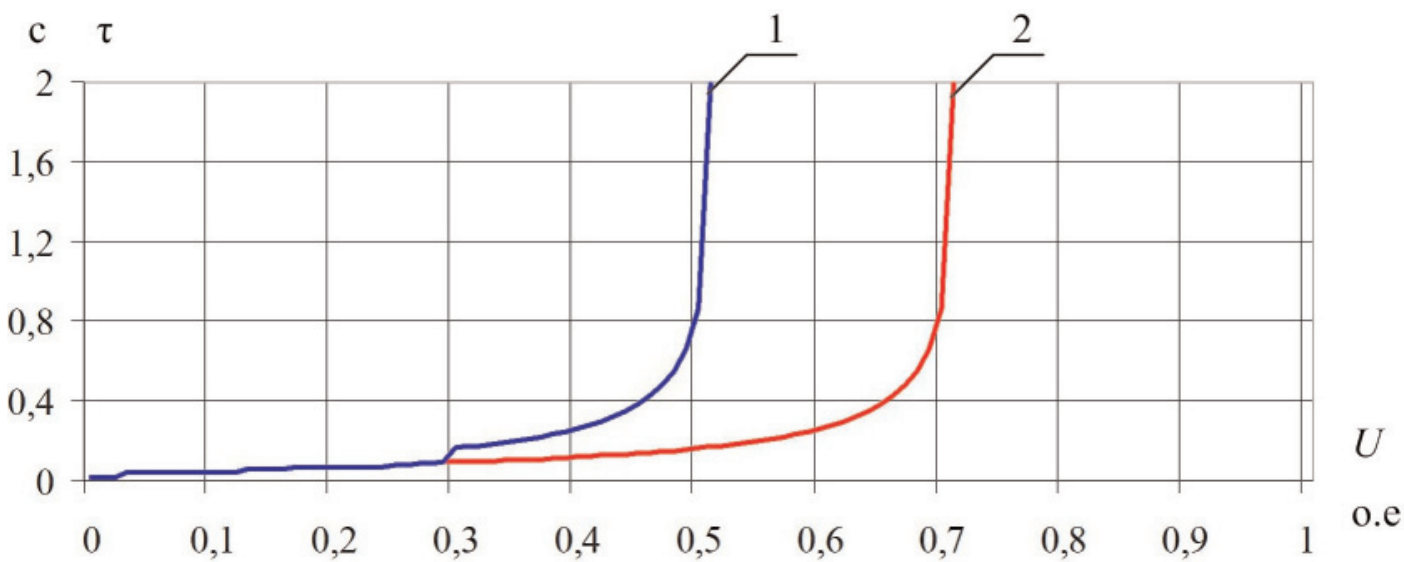

Рис. 3. Границы динамической устойчивости куста скважин: 1 - с применением ДКИн 20\%,2 - исходная

Fig. 3. Dynamic stability curves of the well cluster: 1) when using DVR 20\%,2) original 
Согласно результатам расчета (табл. 2), повышение устойчивости кустов скважин производительностью от 0,5 до 5 т/ч с помощью БАВР и ДКИН не является экономически целесообразным, т. к. коэффициент экономической эффективности капитальных вложений во всех случаях меньше 0,364. Повышение устойчивости узла нагрузки КНС с помощью БАВР показывает, что при производительности КНС $200 \mathrm{~m}^{3} /$ ч коэффициент экономической эффективности капитальных вложений равен 0,58, при производительности $1890 \mathrm{~m}^{3} /$ ч 2,23 , следовательно, чем больше производительность КНС, тем быстрее окупится применение дорогостоящего БАВР. Коэффициент экономической эффективности капитальных вложений в ДКИН для повышения устойчивости узла нагрузки КНС равен 0,59 при производительности $200 \mathrm{~m}^{3} /$ ч и 0,95 - при производительности $1890 \mathrm{~m}^{3} /$ ч.

\section{Заключение}

Для анализа мероприятий по повышению устойчивости узлов электродвигательной нагруз-

\section{СПИСОК ЛИТЕРАТУРЫ}

1. Мищенко И.Т. Скважинная добыча нефти. - М: ФГУП Изд-во «Нефть и газ» РГУ нефти и газа им. И.М. Губкина, 2003. $816 \mathrm{c.}$

2. Меньшов Б.Г., Ершов М.С., Яризов А.Д. Электротехнические установки и комплексы в нефтегазовой промышленности. М.: ОАО «Изд-во «Недра»», 2000. - 487 с.

3. Пупин В.М. Устройства защиты электрооборудования от провалов напряжения длительностью от 10 миллисекунд // Электрооборудование: эксплуатация и ремонт. - 2013. - № 10. C. 23-34.

4. Пупин В.М., Куфтин Д.С., Сафонов Д.О. Новые способы защиты электрооборудования в питающих сетях предприятий при провалах напряжения // Электрооборудование: эксплуатация и ремонт. - 2012. - № 4. - С. 22-28.

5. Нешитой А.С. Инвестиции. - М.: Издательско-торговая корпорация «Дашков и К», 2007. -372 с.

6. СТП 0148463.004-87. Оценка эффективности мероприятий по повышению надежности электрических сетей нефтяных промыслов. - Тюмень: Ротапринт Гипротюменнефтегаза, 1988. $52 \mathrm{c.}$

7. Семенов В.М. Экономика предприятия. - СПб.: Питер, 2008. $416 \mathrm{c.}$

8. Файбисович Д.Л. Справочник по проектированию электрических сетей. - М.: ЭНАС, 2012. - 376 с.

9. Heine P., Pohjanheimomethod P. A method for estimating the frequency and cost of voltage sags / IEEE Transactions on Power Systems. - 2002. - V. 17. - Iss. 2. - P. 290-296.

10. Gupta C.P., Milanovic J.V. Probabilistic Assessment of Financial Losses due to Interruptions and Voltage Sags. Part I // The Methodology. - 2006. - V. 21. - Iss. 2. - P. 918-924.

11. Лосев Ф.А., Сушков В.В. Разработка методики и алгоритмов оценки влияния несимметричных провалов напряжения на устойчивость узла асинхронной электродвигательной нагрузки нефтяных месторождений // Омский научный вестник. 2018. - № 4 (160). - C. 94-98.

12. Jiadai L., Venkata D. Detailed Magnetic Equivalent Circuit Based Real-Time Nonlinear Power Transformer Model on FPGA for Electromagnetic Transient Studies / IEEE Transactions on Industrial Electronics. - 2016. - V. 63. - Iss. 2. - P. 1191-1202. ки целесообразен поликритериальный подход, позволяющий учитывать коэффициент зависимости источников питания, коэффициент запаса устойчивости по напряжению и коэффициент экономической эффективности капитальных вложений. Так, при росте запаса устойчивости по напряжению уменьшается ожидаемое количество отключений узла нагрузки из-за потери устойчивости и требуется увеличивать капитальные вложения в мероприятия по повышению устойчивости.

Для узла нагрузки кустовой насосной станции коэффициент экономической эффективности капитальных вложений уменьшается на $30 \%$ при повышении коэффициента запаса устойчивости по напряжению на 7 \% при помощи динамического компенсатора искажений напряжения.

Коэффициент экономической эффективности капитальных вложений в быстродействующий автоматический ввод резерва для повышения устойчивости узла нагрузки кустовой насосной станции увеличивается в 4 раза при увеличении производительности объекта в 9 раз.

13. Deane J. Modeling the dynamics of nonlinear inductor circuits // IEEE Transactions on Magnetics. - 1994. - V. 30. - Iss. 5. P. 2795-2801.

14. Веников В.А. Переходные электромеханические процессы в электрических системах. - М.: Высшая школа, 1985. - 536 с.

15. Martianov A.S., Sushkov V.V. Ride-through solutions: Classification and comparison // Dynamics of Systems, Mechanisms and Machines (Dynamics). - IEEE, 2016. - P. 1-4.

16. ГОСТ 32144-2013 Электрическая энергия. Совместимость технических средств электромагнитная. Нормы качества электрической энергии в системах электроснабжения общего назначения. - М.: Стандартинформ, 2014. - 18 c.

17. Ершов М.С., Егоров А.В., Анцифоров В.А. Методы оценки надежности и независимости источников питания в системах промышленного электроснабжения // Промышленная энергетика. - 2014. - № 1. - С. 2-6.

18. Мартьянов А.С., Сушков В.В. Обоснование технических решений для повышения динамической устойчивости установок добычи нефти с электрическими центробежными насосами // 0мский научный вестник. - 2017. - № 6 (156). - С. 105-111.

19. Егоров А.В., Новоселова Ю.В. Устойчивость асинхронных многомашинных комплексов при внешних многопараметрических возмущениях // Промышленная энергетика. - 2000. № 11. - C. 24-27.

20. Dynamic Voltage Restore DVR SET. Operating manual. ZIGOR Corporation. URL: http://www.zigor.com/products/power-quality-back-up/zigor-power-avc/ (дата обращения: 03.09.2018).

21. Singh S.K., Srivastava S.K. Enhancement in power quality using dynamic voltage restorer (DVR) in distribution network // International Conference on Innovations in Information, Embedded and Communication Systems. - Tamilnadu, India, 2017. - P. 1-5.

22. Ghosh A., Jindal A.K., Joshi A. Design of a Capacitor-Supported Dynamic Voltage Restorer (DVR) for Unbalanced and Distorted Loads / IEEE Transactions on Power Delivery. - 2004. - V. 19. № 1. - P. 405-413.

23. Rauf A.M., Khadkikar V. An Enhanced Voltage Sag Compensation Scheme for Dynamic Voltage Restorer // IEEE Transactions on Industrial Electronics. - 2015. - V. 62.- Iss. 5.- P. 268-269.

24. Deepa F., Tomson T. Mitigation of voltage sag and swell using dynamic voltage restorer // Annual International Conference on 
Emerging Research Areas: Magnetics, Machines and Drives (AICERA/iCMMD). - Kottayam, India, 2014. - P. 1-6.

25. Ital A.V., Borakhade S.A. Compensation of voltage sags and swells by using Dynamic Voltage Restorer (DVR) // International Conference on Electrical, Electronics, and Optimization Techniques. - Tamilnadu, India, 2016. - P. 1515-1519.

26. Разработка наблюдателя полного порядка с оперативным мониторингом момента сопротивления для погружных асинхронных электродвигателей / А.С. Глазырин, С.Н. Кладиев, К.С. Афанасьев, В.В. Тимошкин, И.Г. Слепнёв, В.И. Поли- щук, H. Sándor // Известия Томского политехнического университета. Инжиниринг георесурсов. - 2018. - Т. 329. - № 2. C. $118-126$

27. Боловин Е.В., Глазырин А.С. Метод идентификации параметров погружных асинхронных электродвигателей установок электроприводных центробежных насосов для добычи нефти // Известия Томского политехнического университета. Инжиниринг георесурсов. - 2017. - Т. 328. - № 1. - С. 123-131.

Поступила 28.11.2018 2.

\section{Информация об авторах}

Лосев $\Phi . A .$, ассистент кафедры электроэнергетики Тюменского индустриального университета.

Сушков B.B., доктор технических наук, профессор кафедры энергетики Нижневартовского государственного университета. 


\title{
POLYCRITERIA APPROACH TO THE ANALYSIS OF ACTIONS FOR INCREASING ELECTROMOTIVE LOADING STABILITY OF THE OIL-EXTRACTING ENTERPRISES
}

\author{
Fedor A. Losev', \\ fedor_los@mail.ru \\ Valery V. Sushkov², \\ sushkovvv@gray-nv.ru \\ 1 Industrial University of Tyumen, \\ 38, Volodarsky Street, Tyumen, 625000, Russia. \\ ${ }^{2}$ Nizhnevartovsk State University, \\ 56, Lenin Street, Nizhnevartovsk, 628600, Russia.
}

Relevance. The oil field technological systems operability directly depends on power supply reliability. The most frequent power supply interruptions are voltage sags which can bring to stability loss of electromotive loading that results in economic losses of the oil-extracting enterprise. Various equipment, for example, dynamic voltage restore and standby power supply, can be used to increase the electromotive loading stability. The correct setup of power supply resumption equipment by automatic connection of the standby power supply at shutdown of the working power supply allows increasing electromotive loading stability, however at the same time it is necessary to consider power supply independence.

The main aim of the research is to develop the polycriteria approach to the analysis of actions for increase in electromotive loading stability of the oil-extracting enterprises considering economic and technical factors.

objects of the research are electromotive loading and equipment for increase in electromotive loading stability.

Methods: mathematical modeling of electromotive loading operating at voltage sags; mathematical modeling of equipment for increase in electromotive loading stability; economic efficiency assessment of equipment for increase in electromotive loading stability.

Results. The authors have proposed the polycriteria approach to the analysis of actions for increase in electromotive loading stability of the oil-extracting enterprises considering economic and technical factors. Voltage stability reserve coefficient, power supplies dependence coefficient and capital investments economic efficiency coefficient were used for efficiency assessment of actions to increase in electromotive loading stability. The analysis of use efficiency of dynamic voltage restore and high-speed automatic transfer equipment for increase in loading stability of sectional pump stations and well clusters was carried out.

\section{Key words:}

Increasing electromotive loading stability, dynamic stability curve, dynamic voltage restore,

high-speed automatic transfer equipment, sectional pump station.

\section{REFERENCES}

1. Mishchenko I.T. Skvazhinnaya dobycha nefti [Well oil production]. Moscow, RGU nefti i gaza im. I.M. Gubkina «Neft i gaz» Publ., 2003. 816 p.

2. Menshov B.G., Ershov M.S., Yarizov A.D. Elektrotekhnicheskie ustanovki i kompleksy $v$ neftegazovoy promyshlennosti [Electrotechnical units and complexes in the oil and gas industry]. Moscow, Nedra Publ., 2000. 487 p.

3. Pupin V.M. Protective devices of electrical equipment from voltage drops with duration from 10 miliseconds. Electric equipment: operation and repair, 2013, no. 10, pp. 23-34. In Rus.

4. Pupin V.M., Kuftin D.S., Safonov D.0. New electric equipment protection methods in power lines of the enterprises at voltage falls. Electric equipment: operation and repair, 2012, no. 4, pp. 22-28. In Rus.

5. Neshitoy A.S. Investitsii [Investment]. Moscow, «Dashkov i K» Publ., 2007. $372 \mathrm{p}$.

6. STP 0148463.004-87. Otsenka effektivnosti meropriyatiy po po vysheniyu nadezhnosti elektricheskikh setey neftyanykh promyslov [Enterprise Standard 0148463.004-87. Effectiveness assessment of actions for increase in reliability of oil fields electrical networks]. Tyumen, Rotaprint Giprotyumenneftegaz Publ., 1988. $52 \mathrm{p}$.

7. Semenov V.M. Ekonomika predpriyatiya [Economy of the enterprise]. St. Petersburg, Piter Publ., 2008. 416 p.

8. Faybisovich D.L. Spravochnik po proektirovaniyu elektricheskikh setey [Projection book of electrical networks]. Moscow, ENAS Publ., 2012. 376 p.
9. Heine P., Pohjanheimomethod P. A method for estimating the frequency and cost of voltage sags. IEEE Transactions on Power Systems, 2002. - V. 17. - Iss. 2. - P. 290-296.

10. Gupta C.P., Milanovic J.V. Probabilistic Assessment of Financial Losses due to Interruptions and Voltage Sags. Part I. The Methodology, 2006, vol. 21, Iss. 2, pp. 918-924.

11. Losev F.A., Sushkov V.V. Technique and algorithms for induction electromotive loading node stability assessment of oil fields at three-phase unbalance voltage sag. Omsk Scientific Bulletin, 2018, no. 4 (160), pp. 94-98. In Rus.

12. Jiadai L., Venkata D. Detailed Magnetic Equivalent Circuit Based Real-Time Nonlinear Power Transformer Model on FPGA for Electromagnetic Transient Studies. IEEE Transactions on Industrial Electronics, 2016, vol. 63, Iss. 2, pp. 1191-1202.

13. Deane J. Modeling the dynamics of nonlinear inductor circuits. IEEE Transactions on Magnetics, 1994, vol. 30, Iss. 5, pp. 2795-2801.

14. Venikov V.A. Perekhodnye elektromekhanicheskie protsessy $v$ elektricheskikh sistemakh [Transient electromechanical phenomena in electrical systems]. Moscow, Vysshaya shkola Publ., 1985. $536 \mathrm{p}$.

15. Martianov A.S., Sushkov V.V. Ride-through solutions: Classification and comparison. Dynamics of Systems, Mechanisms and Machines (Dynamics). IEEE, 2016. pp. 1-4.

16. GOST 32144-2013. Elektricheskaya energiya. Soumestimost tekhnicheskikh sredstv elektromagnitnaya. Normy kachestva elektricheskoy energii $v$ sistemakh elektrosnabzheniya obshchego naznacheniya [State Standard 32144-2013. Electric energy. 
Compatibility of technical means electromagnetic. Quality standards of electric energy in the power supply systems]. Moscow, Standartinform Publ., 2014. 18 p.

17. Ershov M.S., Egorov A.V., Antsiforov V.A. Metody otsenki nadezhnosti i nezavisimosti istochnikov pitaniya $\mathrm{v}$ sistemakh promyshlennogo elektrosnabzheniya [Methods of assessment of reliability and independence of power supplies in the power supply systems]. Industrial power engineering, 2014, no. 1, pp. 2-6.

18. Martyanov A.S., Sushkov V.V. Comparison of ride-through solutions for electric submersible pump units. Omsk Scientific Bulletin, 2017, no. 6 (156), pp. 105-111. In Rus.

19. Egorov A.V., Novoselova Yu.V. Ustoychivost asinkhronnykh mnogomashinnykh kompleksov pri vneshnikh mnogoparametricheskikh vozmushcheniyakh [Asynchronous multiple machine complexes stability in case of external multiparameter interruptions]. Industrial power engineering, 2000, no. 11, pp. 24-27.

20. Dynamic Voltage Restore DVR SET. Operating manual. ZIGOR Corporation. Available at: http://www.zigor.com/products/ power-quality-back-up/zigor-power-avc/ (accessed 3 September 2018).

21. Singh S.K., Srivastava S.K. Enhancement in power quality using dynamic voltage restorer (DVR) in distribution network. International Conference on Innovations in Information, Embedded and Communication Systems. Tamilnadu, India, 2017. pp. 1-5.

22. Ghosh A., Jindal A.K., Joshi A. Design of a Capacitor-Supported Dynamic Voltage Restorer (DVR) for Unbalanced and Distorted
Loads. IEEE Transactions on Power Delivery, 2004, vol. 19, no. 1 , pp. $405-413$.

23. Rauf A. M., Khadkikar V. An Enhanced Voltage Sag Compensation Scheme for Dynamic Voltage Restorer. IEEE Transactions on Industrial Electronics, 2015, vol. 62, Iss. 5, pp. 2683-269.

24. Deepa F., Tomson T. Mitigation of voltage sag and swell using dynamic voltage restorer. Annual International Conference on Emerging Research Areas: Magnetics, Machines and Drives (AICERA/iCMMD). Kottayam, India, 2014. pp. 1-6.

25. Ital A.V., Borakhade S.A. Compensation of voltage sags and swells by using Dynamic Voltage Restorer (DVR). International Conference on Electrical, Electronics, and Optimization Techniques. Tamilnadu, India, 2016. pp. 1515-1519.

26. Glazyrin A.S., Kladiev S.N., Afanasiev K.S., Timoshkin V.V., Slepnev I.G., Polishchuk V.I., Halasz S. Design of full order observer with real time monitoring of load torque for submersible induction motors. Bulletin of the Tomsk Polytechnic University. Geo Assets Engineering, 2018, vol. 329, no. 2, pp. 118-126. In Rus.

27. Bolovin E.V., Glazyrin A.S. Method for identifying parameters of submersible induction motors of electrical submersible pump units for oil production. Bulletin of the Tomsk Polytechnic University. Geo Assets Engineering, 2017, vol. 328, no. 1, pp. 123-131. In Rus.

Received: 28 November 2018.

\section{Information about the authors}

Fedor A. Losev, assistant, Industrial University of Tyumen.

Valery V. Sushkov, Dr. Sc., professor, Nizhnevartovsk State University. 